\title{
Maternal use of folic acid supplements during pregnancy, and childhood respiratory health and atopy
}

\author{
Marga B.M. Bekkers*,\#, Liset E.M. Elstgeest ${ }^{\Uparrow}$, Salome Scholtens ${ }^{+}$, \\ Annemien Haveman-Nies ${ }^{\uparrow}$, Johan C. de Jongste ${ }^{\S}$, Marjan Kerkhof ${ }^{+}$, \\ Gerard H. Koppelman ${ }^{f}$, Ulrike Gehring*, Henriëtte A. Smit** and Alet H. Wijga\#
}

ABSTRACT: Previous studies have suggested possible adverse side-effects of maternal use of folic acid-containing supplements (FACSs) during pregnancy on wheeze and asthma in early childhood. We investigated the association between maternal use of FACSs and childhood respiratory health and atopy in the first 8 yrs of life.

Data on maternal use of FACSs, collected during pregnancy, were available for 3,786 children participating in the Prevention and Incidence of Asthma and Mite Allergy birth cohort study. Questionnaire data on children's respiratory and allergic symptoms were collected annually and allergic sensitisation and bronchial hyperresponsiveness (BHR) were measured at 8 yrs of age.

No overall (from 1 to 8 yrs of age) associations were observed between maternal use of FACSs and (frequent) asthma symptoms, wheeze, lower respiratory tract infection, frequent respiratory tract infection and eczema. Maternal folic acid use was associated with wheeze at 1 yr of age (prevalence ratio 1.20, 95\% $\mathrm{Cl} 1.04-1.39$ ), but not with wheeze at later ages. Pre-natal exposure to FACSs was not associated with sensitisation and BHR.

Apart from a small increased risk of early wheeze, we observed no adverse respiratory or allergic outcomes associated with pre-natal FACSs exposure in our study population.

KEYWORDS: Asthma, atopy, pregnancy, prospective, respiratory tract infections, wheeze

$\mathbf{U}$ se of folic acid supplements before and during pregnancy reduces the risk of neural tube defects and other congenital anomalies [1, 2] and is therefore recommended in many countries, including the Netherlands.

In 2009, two articles were published that raised concerns about possible adverse respiratory health outcomes in young children associated with maternal use of folic acid supplements during pregnancy. In Norway, HÅBERG et al. [3] found that folic acid supplements in the first trimester of pregnancy slightly increased the risk of wheeze and lower respiratory tract infections in children $\leqslant 18$ months of age. In Australia, Whitrow et al. [4] showed that intake of folic acid supplements in late pregnancy (30-34 weeks) was related to an increased risk of asthma at $3.5 \mathrm{yrs}$ and of persistent asthma at 3.5 and 5.5 yrs. An earlier study in mice showed that in utero exposure to a diet supplemented with methyl donors, including folate, was associated with increased DNA methylation, higher levels of T-helper type 2 cytokines and subsequent increased risk of allergic airway disease in offspring [5].
Two recent studies [6, 7] emphasise the need to study these possible harmful effects in older children, as diagnosing asthma at an early age is difficult. We had the opportunity to investigate the effects of folic acid supplementation during pregnancy until the age of 8 yrs and investigate objective markers of asthma symptoms and atopy, i.e. bronchial hyperresponsiveness (BHR) and sensitisation, using data from the longitudinal Prevention and Incidence of Asthma and Mite Allergy (PIAMA) study, with yearly follow-up.

\section{METHODS}

\section{Study design and population}

The study population consisted of children born in the Netherlands in 1996-1997 who participated in the PIAMA birth cohort study. At baseline, 4,146 pregnant females recruited from the general population were included in the study. As 183 (5\%) females were lost to follow-up before any data on the child had been obtained, the study started with 3,963 newborns. A detailed description of the study design has been published previously [8].
AFFILIATIONS

*Institute for Risk Assessment Sciences, Utrecht University, **Julius Centre for Health Sciences and Primary Care, University Medical Center Utrecht, Utrecht,

${ }^{\#}$ Centre for Prevention and Health Services Research, National Institute for Public Health and the Environment (RIVM), Bilthoven, 'Division of Human Nutrition, Wageningen University, Wageningen, ${ }^{+}$Dept of Epidemiology and Bioinformatics, University Medical Center Groningen,

${ }^{\dagger}$ Dept of Paediatric Pulmonology and Paediatric Allergology, Beatrix Children's Hospital, Groningen Research Institute for Asthma and COPD, University Medical Center Groningen, University of Groningen, Groningen, and

${ }^{\S}$ Dept of Paediatrics, Erasmus University Medical Center, Sophia Children's Hospital, Rotterdam, The Netherlands.

CORRESPONDENCE

M.B.M. Bekkers

National Institute for Public Health and the Environment

Postbus 1

Postbak 101

Bilthoven

3720 BA

The Netherlands

E-mail: marga.bekkers@rivm.nl

Received:

June 032011

Accepted after revision: Oct 092011

First published online: Oct 272011

European Respiratory Journal Print ISSN 0903-1936 Online ISSN 1399-3003 
Questionnaires were sent to the parents during pregnancy, at 3 months of age and annually from 1 to 8 yrs of age. Completed parental questionnaires from age 3 months to 8 yrs were available for between 3,270 and 3,943 children in each year.

At the age of 8 yrs, all children still in the study were invited for either a hospital-based examination, including blood sampling and a lung function test, or a community-based examination, including only blood sampling. Blood samples were obtained and specific immunoglobulin (Ig)E to common allergens was measured for 1,713 children; BHR measurements were obtained from 938 children. By the design of the PIAMA study, children of allergic mothers were over-represented in the subgroup of children invited for the hospital-based examination. As a result, the prevalence of maternal allergy was higher $(65 \%)$ in the group of children with data on BHR than in the baseline PIAMA population (31\%).

After exclusion of children with missing data on exposures $(n=91)$ and on all health outcomes at every age $(n=86)$, the study population for analysis consisted of 3,786 (95.5\%) out of 3,963 children. The study protocol was approved by the medical ethics committees of the participating institutes and all parents gave written informed consent.

\section{Outcome assessment}

The annual parental questionnaires contained questions on the child's asthma symptoms and eczema, based on the International Study of Asthma and Allergies in Childhood [9], as well as on respiratory infections. The following seven (dichotomous) outcomes were defined, which were obtained at the ages of 3-8 yrs or 1-8 yrs, respectively, and which pertain to the previous 12 months. 1) Asthma symptoms: at least one attack of wheeze, and/or at least one attack of dyspnoea, and/ or prescription of inhalation steroids for respiratory or lung problems by a medical doctor (at 3-8 yrs). 2) Frequent asthma symptoms: "asthma symptoms" in combination with at least four attacks of wheeze or dyspnoea (subgroup of "asthma symptoms") (at 3-8 yrs). 3) Wheeze: at least one attack of wheeze. 4) Upper respiratory tract infection (URTI): parental report of a doctor-diagnosed flu/serious cold, and/or inflammation of the throat, and/or middle ear infection. 5) Lower respiratory tract infection (LRTI): parental report of a doctordiagnosed bronchitis and/or pneumonia. 6) Frequent respiratory tract infection (fRTI): parental report of at least three respiratory tract infections (bronchitis, pneumonia, flu/serious cold, middle ear infection, sinusitis or inflammation of the throat). 7) Eczema: an itchy rash that came and went on typical eczema sites (the folds of the elbows or behind the knees, around ears or eyes or in front of the ankles).

From the medical examinations at age 8 yrs, the following two outcomes were defined. 1) Sensitisation: serum $\operatorname{IgE} \geqslant 0.70$ $\mathrm{IU} \cdot \mathrm{mL}^{-1}$. 2) BHR: a decrease of $\geqslant 20 \%$ in forced expiratory volume in $1 \mathrm{~s}$ at a cumulative dose of $\leqslant 0.61 \mathrm{mg}$ methacholine bromide (according to the protocol of the European Community Respiratory Health Survey [10]).

\section{Exposure assessment}

In the questionnaire administered during pregnancy, expectant mothers were asked whether or not they used specific vitamin or mineral supplements during pregnancy, including folic acid, pre-natal vitamins (multivitamin supplements especially for pregnant females), multivitamins and vitamin B complex supplements. Four exposure groups of maternal use of folic acid-containing supplements (FACSs) were defined as children whose mothers had used: 1) folic acid-only supplements ("folic acid") $(n=1,998 ; 198$ of them also used pre-natal vitamin supplements and 318 of them also used multivitamin or vitamin B complex vitamins); 2) pre-natal vitamin supplements ("pre-natal vitamin") $(n=287)$ but not folic acid only; 3) multivitamin or vitamin B complex supplements ("multivitamin or vitamin B complex") $(n=199)$ but not folic acid-only or pre-natal vitamins; and 4) no folic acid-only, no pre-natal and no multivitamin/vitamin B supplements at all ("no FACSs"). By "FACSs", we mean all folic acid-containing supplements: folic acid only, pre-natal vitamins, and multivitamin/vitamin B complex supplements $(n=3,786)$. Folic acidonly supplements contain the highest concentration of folic acid $(0.5 \mathrm{mg})$ and pre-natal vitamin supplements typically contain more folic acid $(0.4 \mathrm{mg}, 100 \%$ of the recommended daily allowance for pregnant females) than the multivitamin or vitamin B complex supplements $(0.2 \mathrm{mg}) .80 \%$ of the questionnaires were completed between the 30th and 36th weeks of pregnancy (median 33 weeks of gestation). Use of FACSs by these mothers and by mothers who completed the questionnaire before the 30th week or after the 36th week was similar.

\section{Assessment of covariates}

In the questionnaires, data on several family and child characteristics were assessed. Of these variables, the following were considered potential confounders: sex, birth weight, gestational age, number of older siblings, maternal education (low, intermediate or high), maternal allergy (allergy to house dust mite or pets, hay fever or asthma ever, reported during pregnancy or not), maternal body mass index before pregnancy, maternal smoking during pregnancy (any smoking during the first 4 weeks of pregnancy), maternal use of other vitamin supplements (A, C, D or E) than folic acid-only, prenatal and multivitamin or vitamin B complex supplements, maternal age at child birth, breast feeding duration, smoking in the home by anyone at 1 yr of age (at least once a week), type of day care at $1 \mathrm{yr}$ of age (no care outside the home, small-scale care or day care centre attendance) and region (North, Central or West).

\section{Statistical analyses}

To assess the associations between maternal use of FACSs during pregnancy and the seven respiratory and allergic outcomes in the first 8 yrs of life longitudinally, generalised estimating equations (GEEs) with a log link function were used to obtain prevalence ratios (PRs) [11, 12]. GEEs take into account the correlation between repeated measurements in the same individual. An m-dependent correlation structure was used: $m=5$ for the outcome measure (frequent) asthma symptoms; $\mathrm{m}=7$ for the other outcome measures. An interaction term with age was included in the GEE model to allow the association between maternal use of supplements and the outcomes to vary with age. Log binomial regression analyses were used to obtain PRs for the associations between maternal use of FACSs and sensitisation to allergens and BHR at 8 yrs of age. The PRs were the prevalence of respiratory outcomes in 
the exposed groups (folic acid only, pre-natal vitamins, and multivitamins or vitamin B complex) versus the prevalence of respiratory outcomes in the unexposed group (no FACSs). We also performed these analyses with exposure to any folic acid (three exposed groups together) versus no FACSs. In addition, we divided the children into three groups according to maternal folic acid use in trimesters. Because maternal allergy was considered to be a potential effect modifier, the analyses were repeated, stratified on maternal allergy, and interaction between maternal allergy and maternal use of supplements was tested. Maternal education, maternal allergy, maternal smoking during pregnancy and number of older siblings were included in the statistical models as confounders as the combination of these variables changed some of the associations by $>5 \%$. Furthermore, the prevalence of these variables differed substantially between the four exposure groups. The analyses with frequent asthma symptoms were performed in a group of children with frequent asthma symptoms and children without asthma symptoms. No multivariable analyses could be performed with frequent asthma symptoms because of the small numbers of children with a positive score on this outcome. $\mathrm{p}$ values $<0.05$ were considered statistically significant. All analyses were performed in SAS for Windows version 9.2 (SAS Institute, Cary, NC, USA).

\section{RESULTS}

More than half $(52.9 \%)$ of the pregnant females used folic acid, $13.1 \%$ used pre-natal vitamins, $10.7 \%$ multivitamins and $3.4 \%$

TABLE 1 Characteristics of the study population

\begin{tabular}{|c|c|c|c|c|}
\hline Characteristic & Folic acid & Pre-natal vitamins & $\begin{array}{l}\text { Multivitamins or } \\
\text { vitamin B complex }\end{array}$ & No FACSs \\
\hline Subjects $n$ & 1998 & 287 & 199 & 1302 \\
\hline Males & 1035/1998 (51.8) & $143 / 287(49.8)$ & 97/199 (48.7) & 689/1302 (52.9) \\
\hline \multicolumn{5}{|l|}{ Region } \\
\hline North & 602/1998 (30.1) & $56 / 287(19.5)$ & $56 / 199(28.1)$ & 459/1302 (35.3) \\
\hline Central & $826 / 1998$ (41.3) & $132 / 287(46.0)$ & 73/199 (36.7) & $503 / 1302(38.6)$ \\
\hline West & 570/1998 (28.5) & 99/287 (34.5) & 70/199 (35.2) & $340 / 1302$ (26.1) \\
\hline \multicolumn{5}{|l|}{ Ethnic background } \\
\hline Dutch & 1851/1925 (96.2) & 252/269 (93.7) & 170/187 (90.9) & $1148 / 1236$ (92.9) \\
\hline Western & 32/1925 (1.7) & 6/269 (2.2) & $7 / 187(3.7)$ & 27/1236 (2.2) \\
\hline Non-Western & 42/1925 (2.2) & 11/269 (4.1) & 10/187 (5.3) & $61 / 1236(4.9)$ \\
\hline \multicolumn{5}{|l|}{ Older siblings } \\
\hline 0 & 1095/1986 (55.1) & $118 / 286(41.3)$ & 70/197 (35.5) & $578 / 1290(44.8)$ \\
\hline 1 & $677 / 1986(34.1)$ & $116 / 286(40.6)$ & $79 / 197(40.1)$ & $472 / 1290(36.6)$ \\
\hline 2 & 179/1986 (9.0) & 36/286 (12.6) & 36/197 (18.3) & $155 / 1290(12.0)$ \\
\hline$\geqslant 3$ & $35 / 1986(1.8)$ & $16 / 286(5.6)$ & $12 / 197(6.1)$ & $85 / 1290(6.6)$ \\
\hline Maternal smoking during pregnancy & $277 / 1973(14.0)$ & $49 / 281(17.4)$ & $39 / 198(19.7)$ & 290/1288 (22.5) \\
\hline Prematurity $<37$ weeks & $101 / 1990(5.1)$ & $9 / 285(3.2)$ & $13 / 198(6.6)$ & $63 / 1292(4.9)$ \\
\hline Born by Caesarean section & 163/1976 (8.2) & 23/286 (8.0) & 23/196 (11.7) & $112 / 1275(8.8)$ \\
\hline Allergic mother & $637 / 1998$ (31.9) & $85 / 287(29.6)$ & 77/199 (38.7) & $360 / 1302(27.6)$ \\
\hline Allergic father & 623/1998 (31.2) & 85/287 (29.6) & 66/199 (33.2) & 385/1302 (29.6) \\
\hline \multicolumn{5}{|l|}{ Maternal education level } \\
\hline Low & $370 / 1973(18.8)$ & $82 / 279(29.4)$ & $42 / 197(21.3)$ & $365 / 1276(28.6)$ \\
\hline Intermediate & $817 / 1973(41.4)$ & $116 / 279(41.6)$ & 73/197 (37.1) & $551 / 1276(43.2)$ \\
\hline High & 786/1973 (39.8) & $81 / 279(29.0)$ & $82 / 197(41.6)$ & $360 / 1276(28.2)$ \\
\hline \multicolumn{5}{|l|}{ Paternal education level } \\
\hline Low & 439/1961 (22.4) & $77 / 270(28.5)$ & 42/194 (21.6) & $388 / 1256(30.9)$ \\
\hline Intermediate & 658/1961 (33.6) & $91 / 270(33.7)$ & $70 / 194(36.1)$ & $449 / 1256(35.7)$ \\
\hline High & $864 / 1961$ (44.1) & $102 / 270(37.8)$ & $82 / 194(42.3)$ & $419 / 1256(33.4)$ \\
\hline Smoking in the home at $1 \mathrm{yr}$ of age & 460/1978 (23.3) & $88 / 280(31.4)$ & $68 / 197(34.5)$ & $410 / 1277$ (32.1) \\
\hline \multicolumn{5}{|l|}{ Type of day care at $1 \mathrm{yr}$ of age } \\
\hline No care outside home & $791 / 1978(40.0)$ & $133 / 280(47.5)$ & $90 / 196(45.9)$ & $624 / 1270(49.1)$ \\
\hline Small-scale care & $637 / 1978$ (32.2) & $76 / 280(27.1)$ & $61 / 196(31.1)$ & $396 / 1270$ (31.2) \\
\hline Day care centre attendance & $550 / 1978(27.8)$ & $71 / 280(25.4)$ & 45/196 (23.0) & $250 / 1270(19.7)$ \\
\hline Maternal age yrs & $30.5 \pm 3.8(1983)$ & $30.2 \pm 3.9(285)$ & $31.1 \pm 3.7(197)$ & $30.1 \pm 4.0(1292)$ \\
\hline Maternal BMI before pregnancy $\mathbf{k g} \cdot \mathrm{m}^{-2}$ & $22.8 \pm 3.3(1820)$ & $22.8 \pm 3.3(256)$ & $22.5 \pm 3.4(184)$ & $23.0 \pm 3.5(1130)$ \\
\hline Birth weight $\mathbf{g}$ & $3504 \pm 546(1987)$ & $3525 \pm 553(283)$ & $3569 \pm 601(197)$ & $3504 \pm 536(1278)$ \\
\hline Gestational age weeks & $39.8 \pm 1.7(1990)$ & $39.8 \pm 1.5(285)$ & $39.9 \pm 1.6(198)$ & $39.9 \pm 1.6(1292)$ \\
\hline Breast feeding duration weeks & $15.3 \pm 15.1(1977)$ & $15.1 \pm 15.0(285)$ & $17.3 \pm 15.9(194)$ & $14.3 \pm 14.8(1290)$ \\
\hline
\end{tabular}

Data are present as $\mathrm{n} / \mathrm{N}(\%)$ or mean $\pm \mathrm{SD}(\mathrm{n})$, unless otherwise stated. FACS: folic acid-containing supplement; BMI: body mass index. 
TABLE 2 Prevalence of respiratory and allergic outcomes from 1 to 8 yrs of age

\begin{tabular}{|c|c|c|c|c|c|c|c|c|}
\hline \multirow[t]{2}{*}{ Outcome } & \multicolumn{8}{|c|}{ Age yrs } \\
\hline & 1 & 2 & 3 & 4 & 5 & 6 & 7 & 8 \\
\hline $\begin{array}{l}\text { Asthma } \\
\text { symptoms }\end{array}$ & & & 822/3604 (22.8) & 653/3484 (18.7) & $605 / 3418(17.7)$ & 496/3389 (14.6) & 406/3299 (12.3) & $419 / 3237$ (12.9) \\
\hline $\begin{array}{l}\text { Frequent asthma } \\
\text { symptoms }\end{array}$ & & & $195 / 3586(5.4)$ & $166 / 3470(4.8)$ & $169 / 3409(5.0)$ & 120/3385 (3.6) & $81 / 3290(2.5)$ & 83/3184 (2.6) \\
\hline URTI & 1925/3633 (53.0) & $1975 / 3617$ (54.6) & $1665 / 3577(46.6)$ & $1241 / 3454(35.9)$ & 1247/3352 (37.2) & 1034/3362 (30.8) & $719 / 3249(22.1)$ & 693/3158 (21.9) \\
\hline LRTI & $578 / 3634$ (15.9) & $460 / 3614(12.7)$ & $364 / 3564(10.2)$ & $254 / 3444(7.4)$ & 263/3386 (7.8) & $194 / 3323(5.8)$ & $119 / 3236(3.7)$ & $114 / 3173$ (3.6) \\
\hline fRTI & $512 / 3624(14.1)$ & $532 / 3615(14.7)$ & $418 / 3582(11.7)$ & $343 / 3466$ (9.9) & $341 / 3351$ (10.2) & $260 / 3377(7.7)$ & 138/3230 (4.3) & 122/3179 (3.8) \\
\hline Eczema & $553 / 3605$ (15.3) & $610 / 3629(16.8)$ & 622/3574 (17.4) & 622/3458 (18.0) & $510 / 3301(15.4)$ & $519 / 3352(15.5)$ & 466/3274 (14.2) & $505 / 3166(16.0)$ \\
\hline BHR & & & & & & & & 395/915 (43.2) \\
\hline Sensitisation & & & & & & & & $515 / 1669$ (30.9) \\
\hline
\end{tabular}

Data are presented as n/N (\%). URTI: upper respiratory tract infection; LRTI: lower respiratory tract infection; fRTI: frequent respiratory tract infections; BHR; bronchial hyperresponsiveness.

vitamin B complex supplements; $34.4 \%$ used no FACSs. Some pregnant females used more than one supplement. The use of supplements other than FACSs ranged from $2.3 \%$ for vitamin A or $\mathrm{D}$ to $47.9 \%$ for iron.

Characteristics of the four exposure groups are presented in table 1 . In the group of folic acid-only users, parental education was higher, the prevalence of smoking, both during pregnancy and at 1 yr of age, was lower, and more mothers were expecting their first child than in the group of no FACSs users.

\section{Prevalence of health outcomes}

The prevalence of all outcomes decreased with age with the exception of eczema, which remained stable at around $16 \%$. The prevalence of asthma symptoms decreased from $23 \%$ at $3 \mathrm{yrs}$ of age to $13 \%$ at $8 \mathrm{yrs}$ of age and the prevalence of frequent asthma symptoms from $5.4 \%$ to $2.6 \%$, respectively. The prevalence rates of respiratory and allergic outcomes from 1 to 8 yrs of age are shown in table 2 .

\section{Associations between maternal use of FACSs and respiratory and allergic outcomes}

There were no associations between the use of FACSs and asthma symptoms, frequent asthma symptoms, wheeze, fRTI and eczema. Table 3 shows the overall (over the entire age range) estimates for the associations between maternal use of FACSs during pregnancy and the respiratory and allergic outcomes from 1 or 3 yrs to 8 yrs of age. Borderline significant positive associations were seen between folic acid use and URTIs (PR 1.04, 95\% CI 1.00-1.09) and between pre-natal vitamin use and URTIs (PR 1.07, 95\% CI 0.98-1.17) after adjustment for confounders. Results for any versus no folic acid

TABLE 3 Prevalence ratios of respiratory and allergic outcomes in children of mothers who used folic acid supplements versus children of mothers who did not use folic acid supplements, age 1 to 8 yrs

\begin{tabular}{|c|c|c|c|c|c|c|}
\hline \multirow[t]{2}{*}{ Outcome } & \multicolumn{2}{|c|}{ Folic acid a, $^{\#}$} & \multicolumn{2}{|c|}{ Pre-natal vitamins $\mathbf{s}^{\#,+}$} & \multicolumn{2}{|c|}{ Multivitamins or vitamin B complex ${ }^{\#, s}$} \\
\hline & cPR $(95 \% \mathrm{Cl})$ & $\mathrm{aPR}^{f}(95 \% \mathrm{Cl})$ & cPR $(95 \% \mathrm{Cl})$ & $\operatorname{aPR}^{f}(95 \% \mathrm{Cl})$ & cPR $(95 \% \mathrm{Cl})$ & $\mathrm{aPR}^{f}(95 \% \mathrm{Cl})$ \\
\hline Asthma symptoms & $0.99(0.89-1.11)$ & $1.03(0.92-1.16)$ & $1.03(0.84-1.25)$ & $1.00(0.81-1.23)$ & $1.05(0.82-1.33)$ & $1.02(0.80-1.29)$ \\
\hline Frequent asthma symptoms ${ }^{\# \#}$ & $0.88(0.71-1.09)$ & & $0.88(0.60-1.31)$ & & $1.03(0.66-1.61)$ & \\
\hline Wheeze & $1.00(0.89-1.11)$ & $1.07(0.96-1.20)$ & $1.04(0.85-1.27)$ & $1.04(0.85-1.27)$ & $1.07(0.85-1.35)$ & $1.05(0.83-1.34)$ \\
\hline fRTI & $0.94(0.84-1.06)$ & $1.01(0.89-1.13)$ & $1.07(0.86-1.33)$ & $1.11(0.89-1.39)$ & $1.09(0.87-1.38)$ & $1.13(0.90-1.43)$ \\
\hline Eczema & $0.99(0.89-1.10)$ & $0.98(0.87-1.09)$ & $1.10(0.91-1.32)$ & $1.07(0.89-1.29)$ & $1.06(0.85-1.33)$ & $1.04(0.83-1.30)$ \\
\hline
\end{tabular}

CPR: crude prevalence ratio; aPR: adjusted prevalence ratio; URTI: upper respiratory tract infection; LRTI: lower respiratory tract infection; fRTI: frequent respiratory tract infection. ${ }^{*}$ : using "no folic acid-containing supplements" group $(n=1,302)$ as reference; ": $n=1,998 ;{ }^{+}: n=287 ;{ }^{5}: n=199 ;{ }^{\prime}$ : multivariable associations were adjusted for maternal education, maternal allergy, maternal smoking during pregnancy and number of older siblings; \#\#: no multivariable analyses could be performed due to complexity of the model. *: $p<0.05$. 

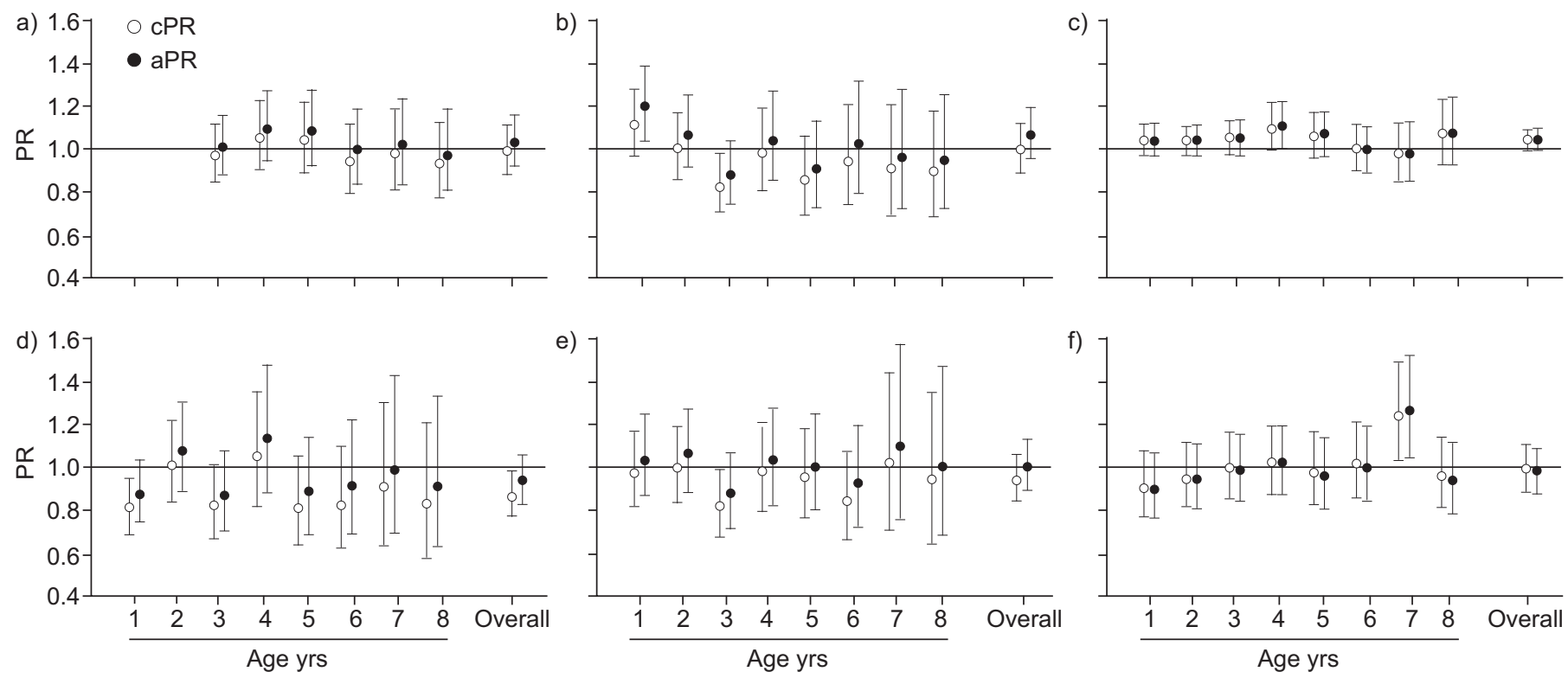

FIGURE 1. Prevalence ratios (PRs) of respiratory and allergic outcomes in children of mothers who used folic acid-containing supplements (FASCs) versus children of mothers who did not use FACSs during pregnancy. Crude PRs (CPRs) and adjusted PRs (aPRs) and 95\% confidence intervals (whiskers) of the associations of the folic acid group versus the no FACSs group and a) asthma symptoms, b) wheeze, c) upper respiratory tract infection, d) lower respiratory tract infection, e) frequent respiratory tract infection and f) eczema from age 1 or 3 yrs to 8 yrs, and the overall effect are shown. Multivariable models were adjusted for sex, maternal education, maternal allergy, maternal smoking during pregnancy and number of older siblings.

exposure were: asthma symptoms, $\beta=1.03$ (95\% CI 0.92-1.15); wheeze, $\beta=1.06$ (95\% CI 0.96-1.19); URTI, $\beta=1.05$ (95\% CI 1.001.09); LRTI, $\beta=0.94$ (95\% CI 0.83-1.06); fRTI, $\beta=1.03$ (95\% CI 0.92-1.16); and eczema, $\beta=0.99$ (95\% CI 0.89-1.10). Overall, the estimates of the associations between any versus no exposure and childhood respiratory outcomes were similar to those of the folic acid-only group.

To investigate the effect of maternal use of FACSs over time, models with an interaction term (age) were used. No clear patterns were observed: PRs fluctuated around 1 and were not statistically significant. The associations between folic acidonly supplement use and respiratory outcomes at each age from birth up to the age of 8 yrs are presented in figure 1 . The GEE analyses showed increased risk of wheeze at the age of $1 \mathrm{yr}$ and increased risk of eczema at the age of $7 \mathrm{yrs}$ (fig. 1). Multivitamin or vitamin B complex use was associated with fRTI at 2 yrs (PR 1.40, 95\% CI: 1.01-1.93) after adjustment. However, the overall estimates were nonsignificant.

\section{Associations between maternal use of FACSs and sensitisation and BHR at $\mathbf{8}$ yrs of age}

Maternal use of FACSs during pregnancy was not significantly associated with sensitisation or BHR at the age of 8 yrs (table 4).

\section{Additional analyses}

Stratified analyses were performed to investigate potential effect modification by maternal allergy. The associations between maternal use of FACSs and respiratory outcomes did not differ between children with and without an allergic mother (no statistically significant associations were observed in either of the two groups). When repeating our analyses separately for FACSs use during the trimesters, we did not find significant associations between FACSs and respiratory outcomes in the children and the estimates were similar in magnitude.

\section{DISCUSSION}

In this study, maternal use of folic acid, pre-natal vitamins, or multivitamin or vitamin B complex supplements during

TABLE 4 Crude and adjusted associations of maternal use of folic acid-containing supplements and sensitisation and bronchial hyperresponsiveness (BHR) at 8 yrs of age

\begin{tabular}{|c|c|c|c|c|c|c|c|}
\hline \multirow[t]{2}{*}{ Outcome } & \multirow[t]{2}{*}{ Subjects $\mathbf{n}$} & \multicolumn{2}{|c|}{ Folic acid $\#$} & \multicolumn{2}{|c|}{ Pre-natal vitamins ${ }^{\#}$} & \multicolumn{2}{|c|}{ Multivitamins or vitamin B complex } \\
\hline & & cPR $(95 \% \mathrm{Cl})$ & $\operatorname{aPR}^{\text {Tा }}(95 \% \mathrm{Cl})$ & cPR $(95 \% \mathrm{Cl})$ & aPR ${ }^{\mathbb{T}}(95 \% \mathrm{CI})$ & cPR $(95 \% \mathrm{Cl})$ & aPR $^{\text {श }}(95 \% \mathrm{Cl})$ \\
\hline Sensitisation at 8 yrs of age & 1650 & $0.91(0.77-1.05)$ & $0.86(0.73-1.01)$ & $0.94(0.71-1.24)$ & $0.94(0.71-1.24)$ & $0.70(0.46-1.05)$ & $0.70(0.46-1.05)$ \\
\hline BHR at 8 yrs of age & 901 & $0.94(0.80-1.10)$ & $0.97(0.82-1.15)$ & $0.97(0.72-1.31)$ & $0.98(0.72-1.34)$ & $0.88(0.60-1.27)$ & $0.94(0.65-1.36)$ \\
\hline
\end{tabular}

cPR: crude prevalence ratio; aPR: adjusted prevalence ratio. ${ }^{*}$ : using "no folic acid-containing supplements" group ( $\left.\mathrm{n}=1,302\right)$ as reference; $\bullet$ multivariable associations are adjusted for maternal education, maternal allergy, maternal smoking during pregnancy and number of older siblings. 
pregnancy was not associated with childhood respiratory health and atopy. We observed no associations between maternal use of FACSs during pregnancy and (frequent) asthma symptoms, wheeze, LRTI, fRTI and eczema up to 8 yrs of age, while maternal use of folic acid-only and pre-natal vitamin supplements were borderline associated with URTIs. In addition, no associations were observed of maternal FACSs use with sensitisation or with BHR at 8 yrs of age. In the analyses of agespecific associations, an increased risk of wheeze was observed at the age of $1 \mathrm{yr}$, but not at later ages.

\section{Strengths and limitations}

Important strengths of the study were the prospective design, the large study population, follow-up to $8 \mathrm{yrs}$ of age with annual data collection and questionnaires available for $83 \%$ of the original PIAMA population. In contrast to previous studies, we had data available on both allergic and respiratory outcome measures, including objective measures, i.e. specific $\mathrm{IgE}$ and $\mathrm{BHR}$, and on a large number of potential confounders. Besides the use of folic acid-only supplements, the use of other supplements containing folic acid was taken into account, which made it possible to distinguish between mothers who used pre-natal vitamin, multivitamin or vitamin B complex supplements during pregnancy and mothers who did not use any FACSs.

However, some limitations should be considered when interpreting our results. We do know which supplements females used at the time they completed the questionnaire, but we do not know what they used in earlier and later stages of pregnancy. Therefore, our exposure variables may not validly distinguish ever-users from never-users. It seems unlikely, however, that strong associations, if they were present, would have been entirely obscured by this form of misclassification.

For the outcome measure of frequent asthma symptoms, no multivariable analyses could be performed because of the small numbers of children with a positive score for this outcome and the resulting complexity of the model. However, adjusted estimates did not differ substantially from crude estimates for the other outcome measures, which makes it likely that this also holds for frequent asthma symptoms.

\section{Findings of other studies}

Only a few studies on this subject have been conducted previously and there are still inconsistencies in the available evidence. In a Norwegian cohort $(n=32,077)$ slightly, but significantly, increased risks of wheeze, LRTIs and hospitalisations for LRTIs up to 18 months of age were found in children exposed to folic acid supplements in the first trimester (relative risk 1.06 (95\% CI 1.03-1.10), 1.09 (95\% CI: 1.02-1.15) and 1.24 (95\% CI 1.09-1.41), respectively) [3]. In our study, the risk of wheeze at the age of 1 yr was also significantly increased in the folic acid group, but we did not find associations at later ages. In addition, we observed a slightly increased risk for URTIs (which were not investigated in previous studies) but not for LRTIs. However, in the Norwegian study, risk of wheeze and LRTIs was only increased if the mother used folic acid supplements in the first trimester of pregnancy and not if she took supplements after the first trimester. Recently, a casecontrol study nested within this Norwegian cohort was performed in which, during the second trimester of pregnancy, maternal folate levels were measured in plasma. An increased risk of asthma was observed for children whose mothers had plasma folate levels in the highest, compared with the lowest, quintile (OR 1.66, 95\% CI 1.16-2.37) [13]. Whereas we found no associations with asthma symptoms at any age, in an Australian cohort $(n=490)$, folic acid supplementation during late pregnancy (30-34 weeks) increased the risk of asthma at $3.5 \mathrm{yrs}$ of age (relative risk 1.26, 95\% CI 1.09-1.47); the association with asthma at 5.5 yrs of age was not statistically significant [4]. In contrast to the Norwegian study, maternal folate intake from supplements in early pregnancy was not associated with increased risk in the Australian study, although the intakes of supplements were substantially higher in early pregnancy (median $667 \mu \mathrm{g}$ per day) than in late pregnancy (median $300 \mu \mathrm{g}$ per day). Similarly, two recent studies that focussed on folic acid supplement use in early pregnancy showed no associations with increased risk of asthma at ages 6 and 7 yrs [6, 7]. Also, in the KOALA birth cohort [6], no associations were found between folic acid supplement use during the whole pregnancy and allergic and respiratory outcomes were found. In line with our results on sensitisation, large British and Dutch birth cohorts observed no association between maternal use of folic acid supplements at several moments during pregnancy and atopy (measured by skin-prick test) in the child at 7 yrs of age $[6,14]$. An increased risk was only observed in the subgroup of children carrying the T allele of the C677T polymorphism of the methylene tetrahydrofolate reductase (MTHFR) gene, if mothers took supplements at 32 weeks, but not if they took supplements at 18 weeks [14]. A Japanese study investigated maternal B vitamin intake during pregnancy from diet (not from supplements) and found no association between dietary folate intake and wheeze and eczema in children aged 16-24 months [15]. For future studies, plasma folate seems the most promising approach to address the questions that emerge from the studies conducted so far, as plasma folate concentration might be a better measure of the child's exposure than folic acid supplement intake.

\section{Conclusion and implications}

Maternal use of FACSs was associated with a small increase in risk of wheeze in the first year of life, but not at later ages, and a marginally increased overall risk of URTIs at 1-8 yrs. No associations were found with asthma symptoms, frequent asthma symptoms, LRTIs, fRTIs, eczema, BHR or sensitisation. Possible adverse side-effects of folic acid supplements need further study as their use by pregnant females is likely to increase. Given the well-established benefits of folic acid supplementation for the prevention of neural tube defects, present evidence of possible adverse side-effects on respiratory and atopic health outcomes does not warrant re-evaluation of current recommendations.

\section{SUPPORT STATEMENT}

Research relating to this manuscript was funded by the Netherlands Organization for Health Research and Development, the Netherlands Organization for Scientific Research, the Netherlands Asthma Fund, the Netherlands Ministry of Spatial Planning, Housing and the Environment, and the Netherlands Ministry of Health, Welfare and Sport.

\section{STATEMENT OF INTEREST}

A statement of interest for G.H. Koppelman can be found at www.erj. ersjournals.com/site/misc/statements.xhtml 


\section{REFERENCES}

1 Lumley J, Watson L, Watson M, et al. Periconceptional supplementation with folate and/or multivitamins for preventing neural tube defects. Cochrane Database Syst Rev 2000; 2: CD001056.

2 Blencowe H, Cousens S, Modell B, et al. Folic acid to reduce neonatal mortality from neural tube disorders. Int J Epidemiol 2010; 39: Suppl. 1, i110-i121.

3 Håberg SE, London SJ, Stigum H, et al. Folic acid supplements in pregnancy and early childhood respiratory health. Arch Dis Child 2009; 94: 180-184.

4 Whitrow MJ, Moore VM, Rumbold AR, et al. Effect of supplemental folic acid in pregnancy on childhood asthma: a prospective birth cohort study. Am J Epidemiol 2009; 170: 1486-1493.

5 Hollingsworth JW, Maruoka S, Boon K, et al. In utero supplementation with methyl donors enhances allergic airway disease in mice. J Clin Invest 2008; 118: 3462-3469.

6 Magdelijns FJ, Mommers M, Penders J, et al. Folic acid use in pregnancy and the development of atopy, asthma, and lung function in childhood. Pediatrics 2011; 128: e135-e144.

7 Martinussen MP, Risnes KR, Jacobsen GW, et al. Folic acid supplementation in early pregnancy and asthma in children aged six. Am J Obstet Gynecol 2012; 206: 72.

8 Brunekreef B, Smit J, de Jongste J, et al. The prevention and incidence of asthma and mite allergy (PIAMA) birth cohort study: design and first results. Pediatr Allergy Immunol 2002; 13: Suppl. 15, 55-60.

9 Asher MI, Keil U, Anderson HR, et al. International Study of Asthma and Allergies in Childhood (ISAAC): rationale and methods. Eur Respir J 1995; 8: 483-491.

10 Burney PG, Luczynska C, Chinn S, et al. The European Community Respiratory Health Survey. Eur Respir J 1994; 7: 954-960.

$11 \mathrm{Lu} \mathrm{M}$, Tilley $\mathrm{BC}$. Use of odds ratio or relative risk to measure a treatment effect in clinical trials with multiple correlated binary outcomes: data from the NINDS t-PA stroke trial. Stat Med 2001; 20: 1891-1901.

12 Spiegelman D, Hertzmark E. Easy SAS calculations for risk or prevalence ratios and differences. Am J Epidemiol 2005; 162: 199-200.

13 Håberg SE, London SJ, Nafstad $\mathrm{P}$, et al. Maternal folate levels in pregnancy and asthma in children at age 3 years. J Allergy Clin Immunol 2011; 127: 262-264.

14 Granell R, Heron J, Lewis S, et al. The association between mother and child MTHFR C677T polymorphisms, dietary folate intake and childhood atopy in a population-based, longitudinal birth cohort. Clin Exp Allergy 2008; 38: 320-328.

15 Miyake Y, Sasaki S, Tanaka K, et al. Maternal B vitamin intake during pregnancy and wheeze and eczema in Japanese infants aged 16-24 months: the Osaka Maternal and Child Health Study. Pediatr Allergy Immunol 2010; 21: 22-28. 